


\section{Telemedicine and home care: controversies and opportunities}

\section{Educational aims}

1 To motivate the interest of chest physicians in a new approach to delivering medical care.

1 To define the different types of telemedicine: telecare and telesurveillance.

1 To provide information on the main clinical results in this field and give examples of case studies.

1 To provide details on the recommendations and limitations of telemedicine programmes.

\section{Summary}

This review focuses on the prospects, and possible drawbacks, of a new innovative instrument of care known as "home telehealth", "telecare" or "telemedicine". The main results from utilising telemedicine in respiratory diseases (asthma and chronic obstructive pulmonary disease (COPD) as the illustrative conditions) are presented. A principal goal of telemedicine is to improve access to healthcare services. During this process, savings in time and travel costs should be achieved, thereby rationalising access to medical care. The field of telemedicine is relatively new and expanding. In order to establish evidencebased guidelines for the design and implementation of disease management plans that employ telemedicine, further research is required. Telemedicine is not simply "technology" but an innovative medical approach (based more on a dedicated healthcare team than on high-tech instruments) that will help the medical team to care for patients and their families. In the future, it is hoped that telemedicine will form a valuable part of the disease management process, because, when used intelligently, home telehealth should supplement conventional delivery techniques and not replace them.

Telemedicine is a useful application of information and communication technologies, which may deliver high-quality healthcare senvices regionally, nationally or globally. Telemedicine enables remote, isolated and rural areas to obtain clinical support from distant hospitals and medical systems with a higher level of medical expertise. With advances in electronics, communications, software and hardware, it has become possible to transmit many vital parameters to hightech healthcare centres $[1,2]$. Telemedicine is a combination of medical expertise, medical equipment, computer hardware and software, and communication technology, by which a patient can be examined, investigated, monitored and treated by a medical expert from a distance [2].
M. Vitacca 1

S. Scalvini2 2

A. Spanevello 3

B. Balbi ${ }^{4}$

1 Divisione di Pneumologia, and 2Servizio di Telemedicina, Fondazione S. Maugeri, IRCCS, Gussago/Lumezzane, and ${ }^{3}$ Divisione di Pneumologia, Fondazione S. Maugeri, IRCCS, Cassano, and ${ }^{4}$ Divisione di Pneumologia, Fondazione $S$. Maugeri, IRCCS, Veruno, Italy.

Correspondence:

M. Vitacca

Divisione di Pneumologia Fondazione S. Maugeri IRCCS Gussago/Lumezzane

Via Pinidolo 23

25064 Gussago (BS)

Italy

Fax: 390302521718

E-mail:mvitacca@fsm.it

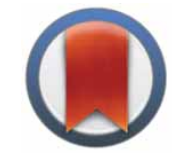

CrossMark $\leftarrow$ click for updates 


\section{Definition of telemedicine}

"Tele" is a Greek word meaning "distance" and "mederi" is a Latin word meaning "to heal". Time Magazine described telemedicine as "healing by wire". Hence, telemedicine is "medicine practiced at a distance". In 1998, it was defined by the World Health Organization as "the delivery of healthcare services, where distance is a critical factor, by all healthcare professionals using information and communication technologies for the exchange of valid information for diagnosis, treatment and prevention of disease and injuries, research and evaluation, and for the continuing education of healthcare providers, all in the interest of advancing the health of individuals and their communities". It can also be defined as "organisation and integration of information technology in such a way that resources outside the local organisation can be used systematically in the activities of health services" $[1,2]$.

\section{Classification}

Telemedicine can be classified on the basis of the mode of operation or type of application [1, 2]. According to the mode of operation, it can be divided into two groups: 1) real-time (interactive) mode, and 2) store-and-forward mode. In the real-time interactive mode, the patient is present with an attending physician or paramedical personnel, and a specialist is present at a remote medical centre. In the store-and-forward mode, all relevant information (data, graphics, images, etc.) is transmitted electronically to the specialist. The diversity of the telematic intervention used ranges from simple telephone follow-up to sophisticated and comprehensive management programmes composed of multiple team members. "Telecare" is a form of telemedicine, and refers to medical/nursing management interventions made over the telephone. "Transtelephonic monitoring" refers to the transmission of physiological variables over existing telephone lines to a network server, which is then displayed on a conventional personal computer. The transmission of physiological variables (heart rate, blood pressure, weight, etc.) and biological signals (ECG trace, oximetry, spirometry, etc.) provide objective data, which may indicate the need for medical/nursing interventions that are subsequently made over the telephone.

\section{Application}

Various telemedicine approaches have been reported in the literature. As telecommunication technology has advanced and costs have declined over the past decade, there has been a steady growth in telemedicine. Much of this

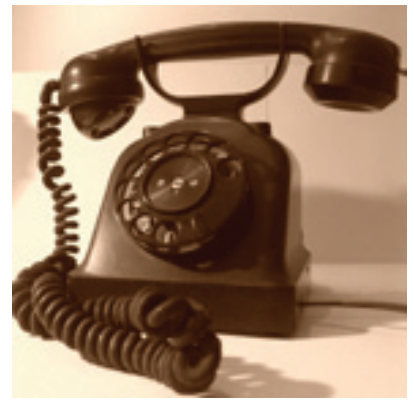

\section{History of telemedicine}

The birth of telemedicine occurred when long-distance healthcare services came into existence. The first telephone technology was invented in 1876 by Alexander Graham Bell. After the 1880s, physicians experimented with telecommunication technologies in managing medical practices. However, telephone technology was not fully developed until World War I, with the possibility of long-distance transmission of medical information being established in the 1900s. Willem Einthoven developed the long-distance broadcast of graphic data in 1905 and later widely utilised it in transmitting electrocardiographs at the Presbyterian Hospital of New York in 1910. Moreover, long-distance diagnoses had been used in communications between rural physicians and urban hospitals after World War I.

A well-known telemedicine project was established in the 1960s when the University of Nebraska transmitted neurological examinations across its campus for medical purposes. The university also set up a telemedicine link 112 miles away with the state hospital (Norfolk State Hospital, Nebraska state), which provided speech therapy, neurological examinations, diagnosis for difficult psychiatric cases, case consultations, research seminars, and education and training.

The National Aeronautics and Space Administration (NASA) pioneered the satellite communication required to demonstrate telemedicine services in the 1960s. NASA tested satellite-based communication to remote areas where it was difficult to receive radio waves, including spacecraft, aircraft, mountains and valleys. From the early 1970s to the late 1980s, digital communication techniques became common, and telemedicine improved in quality, accuracy, convenience and cost.

Between 1995 and 1997, the US Department of Defense established telemedicine links via satellite that connected the battlefield with several different medical centres in Europe, the USA and nearby aircraft carriers. The system reduced referral times and saved lives. The capabilities of this project included computerised medical records, fullmotion remote video consultation between medical units and tertiary care facilities, forward delivery of laboratory and radiology results and prescriptions, digital diagnostic devices (such as ultrasound and film-less teleradiology), and medical command and control technologies. 


\section{Home telenursing}

A new type of nurse involved in telemedicine programmes is a "nurse tutor" or "nurse specialist".

The following steps need to be implemented for this role.

1. Nurses or ancillary staff involved in telesurveillance need specific education and training on respiratory diseases, use of drugs, devices, oxygen therapy, use of mechanical ventilation and tracheotomy assistance.

2. These team members need to be able to use dedicated flowcharts on names, dosages and the timetable of the medicines prescribed.

3. The nurses need competencies to interview patients on clinical conditions, comorbidity, quality of sleep, nighttime problem list, educational needs and monitoring instruments.

growth, however, has been in the form of feasibility studies and pilot trials. Crucially, the main problem in telemedicine is not a lack of technology; instead, it is the organisational problem of knowing how best to take advantage of the technology. In a recent review on the costeffectiveness of telemedicine [3], there was no evidence to suggest that telemedicine is a costeffective means of delivering healthcare [4]. The same review emphasised the need for better designed and conducted trials in particular areas, such as chronic heart failure. In 2001, WootTon [5] published a clinical review on telemedicine and distinguished different types of possible application, as follows.

\section{Home telenursing}

Over the past decade, there has been considerable interest in the possibility of using telemedicine as an aid in home nursing. Nurse telephone triage lines have been running for over 10 years. The nurses who answer the telephones are specially trained and use algorithms on computers to help guide the conversation to a particular conclusion. Little is known about the effectiveness of triaging by telephone, probably because these schemes are not easy to assess. The data in the literature show a benefit in terms of reduced hospital admissions and cost savings $[2,5,6]$, but transposing the benefits to a wider population outside of a trial is not easy. Diagnosis and even prescribing by nurses remains an area of controversy that has not yet been fully established. However, in particular areas, such as chronic disease, nurses manage patients in close collaboration with general practitioners (GPs) and specialists, as a part of an interdisciplinary management process. Various feasibility studies into a range of different kinds of technology have been driven by the hope that chronically ill patients can be provided with either lower-cost or higher-quality care than that which is provided by the traditional home visit. Although these studies indicate that patient satisfaction is not a problem, little hard evidence on costeffectiveness has been obtained [6].

\section{Electronic referrals to specialists and hospitals}

Electronic referrals can be dealt with by the hospital staff without the patient needing to attend the outpatient clinic, either by electronic messages or by arranging a teleconsultation by video link. Another possible application for this model is the interaction between small or peripheral hospitals with larger and super-specialised hospitals. In this case, advice to doctors is provided by a panel of consultants who are not on site and answer via a teleconsultation. This scheme is likely to be costeffective $[7,8]$.

\section{Teleconsulting between GPs and specialists}

Effective teleconsultation often takes place after the transmission of biological signals between experienced specialists and the GP who knows his patients very well; this requires the reciprocal reliance and compliance of GPs and specialists in managing problems, and also in following common diagnostic and therapeutic protocols. While augmenting this professional relationship, telemedicine can aid the appropriateness of hospital admissions, referrals to the emergency department, diagnostic test requests and chronic disease home management $[2,9,10]$.

\section{Call centres and online health}

The growth in telephone call centres that provide health information and advice shows that there is a demand from the public for these services. Many call centres, such as the National Health Service (NHS) Direct in the UK, try to triage callers into those requiring emergency treatment, those who can be referred to primary care, and those who can be advised to treat themselves. Although there is reasonable evidence that these services are safe, little evidence exists to show that they reduce demand and costs for the NHS. The mandatory "quality aims" and questions that telemedicine programmes should address are: is it safe, feasible, effective, sustainable, timely, efficient, equitable, and patient centred? 
Table 1 summarises the mandatory prerequisites for starting a telemedicine programme, and table 2 summarises the useful indicators and measures of outcome when a new telecare programme is proposed in a clinical setting.

\section{Telepneumology}

Very few studies on telepneumology have been published; most have focused on asthmatic patients to test routine review of therapy by telephone or on COPD patients to address the potential impact of telemedicine on improving the sickness impact profile scores, adherence to treatment regimen, mortality rates and healthcare utilisation, e.g. home visits by nurses and emergency admissions.

\section{Table 1 Prerequisites for a home telemedicine programme}

Patients
Informed written consent
Assessment to determine access to utilities and safety for installation of equipment
Patients or caregiver must demonstrate their ability to use and maintain equipment
Providers
A physician usually needs to be involved in the plan
Continuous documentation of calls
Communication between call centre, nurse/ancillary team member and physicians
Written instructions to patients on who to contact in case of problems
Technology
Technology should be based on the patient's clinical and functional needs
Continuous check for accuracy
Safety instructions should be given to patients

\section{Table 2 Indicators and measures of outcome in telemedicine programmes}

\begin{tabular}{ll} 
Indicators & Measures \\
Quality of life & Morbidity \\
Quality of care & Number of hospitalisations, emergency department \\
& visits, GP visits and pulmonologist visits \\
Timelines & Length of stay, time to see a healthcare worker, time to \\
& have a consultation, distance to the nearest healthcare \\
& facility \\
Availability & Distance to nearest facility \\
Health status & Morbidity, client assessment, health status tools \\
Equipment & Installation, costs, service charge \\
Patient/itinerary clinician travel & Cost per trip to healthcare centre \\
Communication & Line fees, long distance connection, data transmission \\
& costs, mobile phone usage \\
Administrative & Expenses, supplies \\
Staffing & Salaries, fees for service, hospital personnel costs \\
Rate of use & Number of consultations per month \\
Convenience & Number of trips avoided \\
Client satisfaction & Global impressions \\
Technological performance & Physical presence, confidentiality \\
\hline Table modified from [1]. &
\end{tabular}

\section{Asthma}

In particular, telemonitoring of asthma has been assessed in paediatric patients with quite similar results in all the studies, in terms of improvement in asthma symptom control and in quality of life [11]. The study by PInNock et al. [11] to determine whether routine review by telephoning patients with asthma improved access and was a good alternative to face-to-face review in general practice showed that neither resulted in an improvement in asthma-related quality of life or morbidity, so it may be tempting to conclude that routine reviews of asthma patients are ineffective. In spite of the results, the study could serve to describe a typical future telephone intervention; telephone reviews were of shorter duration than the face-to-face consultations, although the content was similar, apart from the assessment of practical procedures such as peak flow measurements. The distribution of the timing of consultations suggest that the face-to-face consultations may have been paced to use the available 15-, 20- or 30-minute appointments, whereas a telephone review can take as short or as long a time as needed. Time may be saved during a telephone review, as patients do not have to enter or leave the room, and computer templates and medical records can be completed during the course of the consultation.

The nurses who undertook the reviews observed that the telephone consultations felt more "focused", which may reflect the recognised tendency for telephone interactions to be more goal oriented, with fewer digressions and faster archiving of shared tasks. Patient satisfaction was equally high with both modes of consultation. The nursing-care satisfaction questionnaire included a domain that reflects "perceived time", and it is reassuring that, despite the shorter duration of telephone consultations, there was no evidence of dissatisfaction with the time spent.

In 31 asthmatic patients, FinKELSTEIN et al. [12] demonstrated that spirometry selftesting during telemonitoring is valid and comparable with those tests collected under the supervision of a trained medical professional. Importantly, the same study also emphasised the idea that Internetbased home asthma telemonitoring can be successfully implemented in a group of patients with no computer background [12]. CHAN et al. [13] demonstrated that an Internetbased, store-and-forward video assessment of 10 asthmatic children appeared effective and well accepted. A total of 321 videos of inhaler use 
and 309 videos of peak flow meter use were submitted. Inhaler technique scores, patients' quality-of-life scores and satisfaction improved significantly. No emergency department admissions were recorded during the study [13]. The feasibility of a remote monitoring system for asthmatic patients to use in their own homes following discharge from hospital after an acute exacerbation has also been tested in 84 subjects with success [14].

\section{COPD}

COPD is one of the most common chronic illnesses in the adult population and is responsible for 13\% of hospitalisations [15]. Appropriate long-term management of COPD, including pharmacotherapy, patient education and pulmonary rehabilitation, combined with early recognition of exacerbations and timely treatment, can reduce morbidity and acute health-senvice utilisation [16]. Unfortunately, inadequate patient education, lack of patient compliance to medication, and the failure to detect early symptoms of an exacerbation before the patient requires emergency department admission and/or hospitalisation are common problems in the management of patients with COPD [17].

It has been recognised that interventions that enhance symptom self-monitoring by COPD patients and increase their understanding of COPD therapy may reduce the occurrence of COPD-related hospitalisation and use of other acute healthcare services [18]. Until this point, such interventions have involved the use of timeintensive education and patient monitoring by nurses and other personnel, and have been expensive and inconvenient for patients, as they typically require patients to attend a medical facility on a regular basis [18-21]. For COPD patients, the role of telecare was tested in the management of exacerbations of the disease in the home [22], and the costeffectiveness of telemedicine for the delivery of outpatient pulmonary care to a rural population [23]. In other studies, COPD patients have been included as chronic patients similar to chronic heart failure patients, as in the Kaiser Permanente Organisation scheme [24].

Although a telecommunications system has not yet been employed in the management of patients with COPD, a few published trials have addressed the potential impact of interventions that improve the monitoring of patients with COPD and adherence to their treatment regimen [19-22]. Despite certain methodological

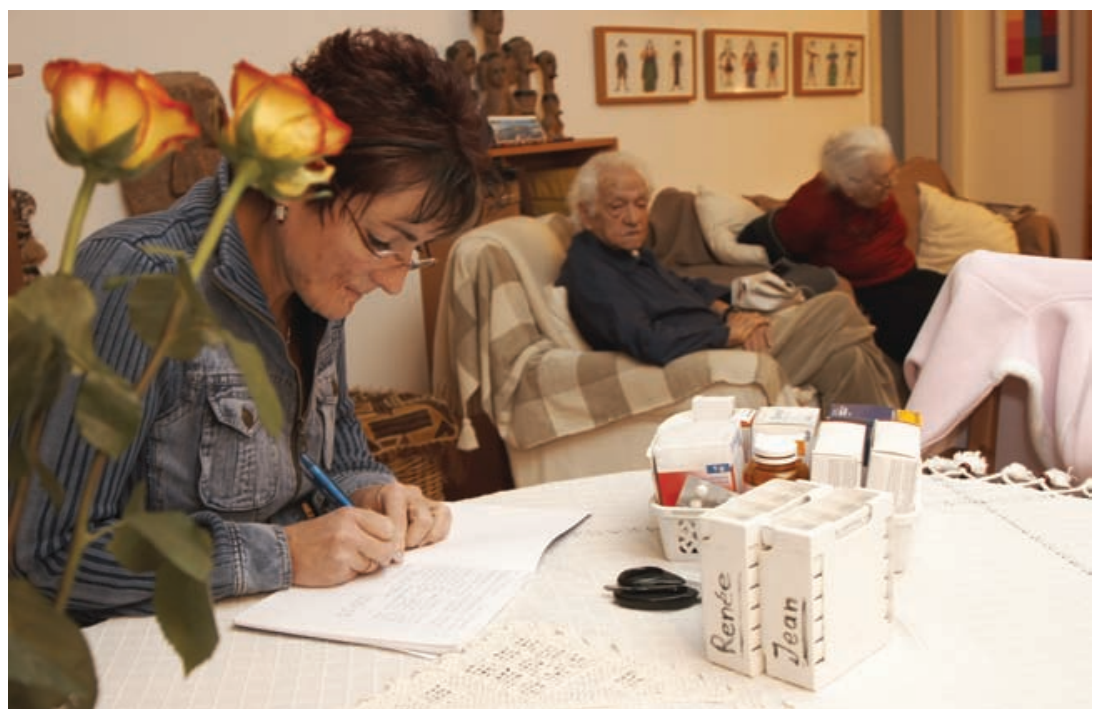

limitations, including small sample size, these studies have shown that COPD monitoring interventions appear to reduce either healthcare utilisation or mortality rates in COPD patients. A reduction of $50 \%$ in costs for emergency services and total expenses for general practice was achieved in a randomised controlled trial, which evaluated an in-patient education programme for patients admitted to hospital with COPD [19].

Similar results were reported by LITLEOHNS et al. [20] in a randomised controlled trial concerning the effectiveness of an outpatient respiratory care worker who provided patient education, symptom monitoring and treatment monitoring, and who served as a liaison between primary care providers and hospital services. In the intervention group, there was a reduction in mortality rate, sickness impact profile scores and the indices of impairment. COCKCROFT et al. [21] conducted a randomised controlled trial in patients with advanced COPD, evaluating the effectiveness of a monthly home visit by a nurse who provided patients with education in adherence with therapeutic regimens and the early self-detection of clinical deterioration. Cost and effectiveness data from the Milwaukee and Iron Mountain Veterans Affairs Medical Centers (VAMC) telepulmonary programme [23] were collected for a period of 1 year. The costeffectiveness analysis was conducted from a societal perspective. Average and incremental costeffectiveness ratios were calculated together with sensitivity analysis. Telemedicine was found to be more costeffective (US\$335 per patient per year) compared to routine care (US\$585 per patient per year) and on-site care (US\$1,166 per patient per year). Sensitivity analysis revealed that the cost 
effectiveness of telemedicine was sensitive to changes in the number of patients, probability of successful telemedicine consultation, telemedicine equipment cost, utility of telemedicine, and percentage effort expended by the on-site pulmonary physician. Telemedicine is a costeffective alternative for the delivery of outpatient pulmonary care for rural populations with limited access to subspecialty services. Costeffectiveness of telemedicine is related to three major factors: cost sharing, i.e. adequate patient volume and sharing of telemedicine infrastructure amongst various clinical users; effectiveness of telemedicine in terms of patient utility and successful clinical consultations; and indirect cost savings accrued by reducing the cost of patients' lost productivity.

The Kaiser Permanente Organisation [24] recently reported the first formal randomised controlled trial of home videophones. In this trial, patients newly diagnosed with a chronic condition were nursed at home. Patients in the intervention group were equipped with home videophones, an electronic stethoscope and a digital blood pressure monitor. Over 18 months, patients in the telemedicine group received $17 \%$ fewer home visits by nurses than the control group and the average cost of care in this group was $27 \%$ less than in the control group. The patients receiving telemedicine were pleased with the equipment and were nursed as effectively as the control group.

Recently, 155 COPD patients with exacerbations were recruited after hospital discharge and randomly assigned to either integrated care (individually tailored care plan upon discharge shared with the primary care team, as well as accessibility to a specialised nurse case manager through a web-based call centre) or usual care. The integrated care intervention was able to decrease hospitalisations for exacerbations [25].

Case study 1 below shows a case report of a COPD patient treated with telecare during a relapse of his disease.

\section{Figure 1}

Oxygen saturation measured by pulse oximetry (orange; $\mathrm{Sp}, \mathrm{O}_{2}$ ) and heart rate (blue; HR) trend during the relapse.

\section{Figure 2}

$\mathrm{Sp}_{\mathrm{O}} \mathrm{O}_{2}$ (orange) and $\mathrm{HR}$ (blue) trend during the recovery.

\section{Case study 1}

A patient affected by a severe COPD and hypoxaemic chronic respiratory insufficiency (CRI) participating in a telemededicine programme requested telephone contact as he was experiencing features of disease relapse with saturimetric worsening. He sent saturimetric tracings via a modem system, which confirmed the relapsing situation (figure 1). Over a period of 15 days, after seven telephone calls scheduled by a nurse tutor, three requests for saturimetric tracing, a change in drug therapy from a pulmonologist and contact with a $\mathrm{GP}$, the patient recovered from his acute event. A new saturimetric tracing confirmed a new clinical stability (figure 2).

It should be noted that clinical and instrumental monitoring via a telematic programme for patients affected by severe COPD and hypoxaemic CRI with home care of a severe relapse is feasible if a well-conducted programme of telemedicine, daily nurse input, saturimetric tracing and a pulmonological second opinion are available.
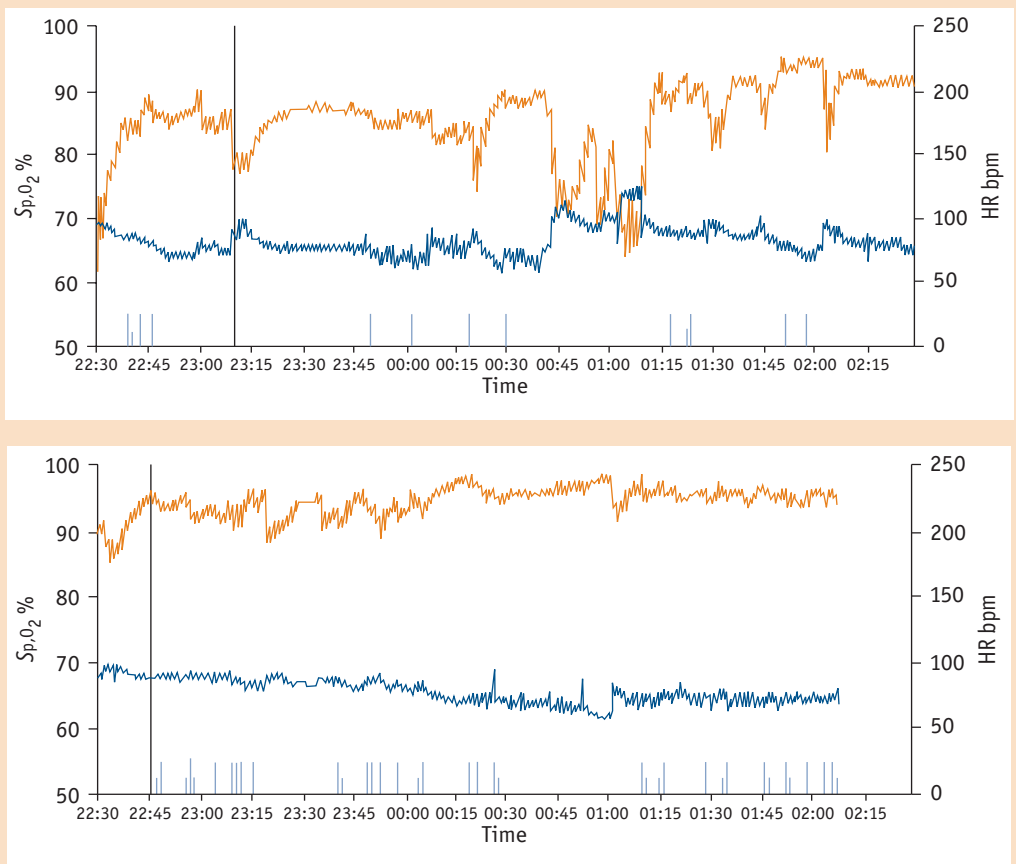


\section{Case study 2}

A 68-year-old patient affected by severe chronic respiratory failure (CRF) due to amyotrophic lateral sclerosis (ALS) received mechanical ventilation (MV) as life-support therapy in the terminal phase of the disease. She was admitted to the current authors' telemedicine programme after intensive care unit discharge. The programme consisted of organised telephone calls, pulse oximetry on demand, a web-based call centre with a specialised nurse and the possibility for the nurse to refer to a doctor. She died 11 months after admission onto the telemedicine programme.

During the period of the programme, she had no hospitalisations, emergency department visits or urgent calls to the GP. She and her caregivers used programmed phone calls (2.7 \pm 0.9 per month) and patientrequested extra phone calls ( $0.8 \pm 0.1$ per month) with regard to counselling to avoid unnecessary hospital admission, coordination of care for the "final" worsening event and resuscitation manoeuvres. The team carried out the following actions: interactive GP consultation, change in medical treatment schedule, sedative or morphine prescription, solving of MV complications, solving clinical problems, obtaining pulmonologist's second opinion, pulse oximetry requests, discussion about the progress of illness, and coordination with the home nurse service. The patient had not completed advance written directives related to end-oflife care.

It should be noted that telemedicine may: 1) improve communication between the hospital staff and a family member; 2) improve the way in which they are informed; 3 ) improve dignity and level of care (receiving adequate pain and symptom management, achieving a sense of control, relieving burden, avoiding inappropriate prolongation of life, and solving medical and MV technical problems); and 4) rationalise the hospital and healthcare necessity in this particular period of life.

\section{Other respiratory diseases}

The application of telemedicine in patients affected by obstructive sleep apnoea to deliver home-based education, provide an alternative to the sleep laboratory and to monitor continuous positive airway pressure application has been also studied with encouraging results [26, 27]. A promising new field of application is the development of a telehospice service as one in which telemedicine is used to provide hospice care in the home. DoolitTlE [28] measured costs for traditional hospice care, as well as those associated with launching and operating a telehospice service, demonstrating a great cost saving when visits were conducted with this new organisation (see Case study 2 above).

A previous study has shown that integrating home telehealth (units that used standard phone lines to communicate with the hospital) with the healthcare institution's electronic database significantly reduces resource use and improves cognitive status, treatment compliance and stability of chronic disease for 104 homebound elderly patients with common complex co-morbidities [29].

\section{CRI}

For 12 months, MAIOLO et al. [30] studied 23 patients with CRF who carried out a fixed transmission of $\mathrm{Sp}_{1} \mathrm{O}_{2}$ twice a week. A reduction in hospital admissions and home relapses of 50 and $55 \%$, respectively, was demonstrated. The use of telemedicine to improve the care of patients on home MV has been demonstrated by MIYASAKA et al. [31], showing that videoconferencing results in an improvement in the quality of paediatric home ventilatory care.

Recently, the current authors demonstrated the feasibility of a telepneumology programme from the home in severe CRF patients with a miscellaneous diagnoses necessitating home MV [31]. The telemedicine team used an $\mathrm{Sp}_{1} \mathrm{O}_{2}$ recording system for titration of oxygen, MV settings and stabilisation of relapses with an optimal level of interaction between nurse, doctor, family and GP. Transmission by modem was often time consuming and difficult for the patient and caregiver (see Case study 1 (page 154) and case study 3 (page 156) for examples) [31]. Figure 3 illustrates the operative chain for the telepneumology programme.

\section{Figure 3}

Operative chain for the telepneumology programme [31].

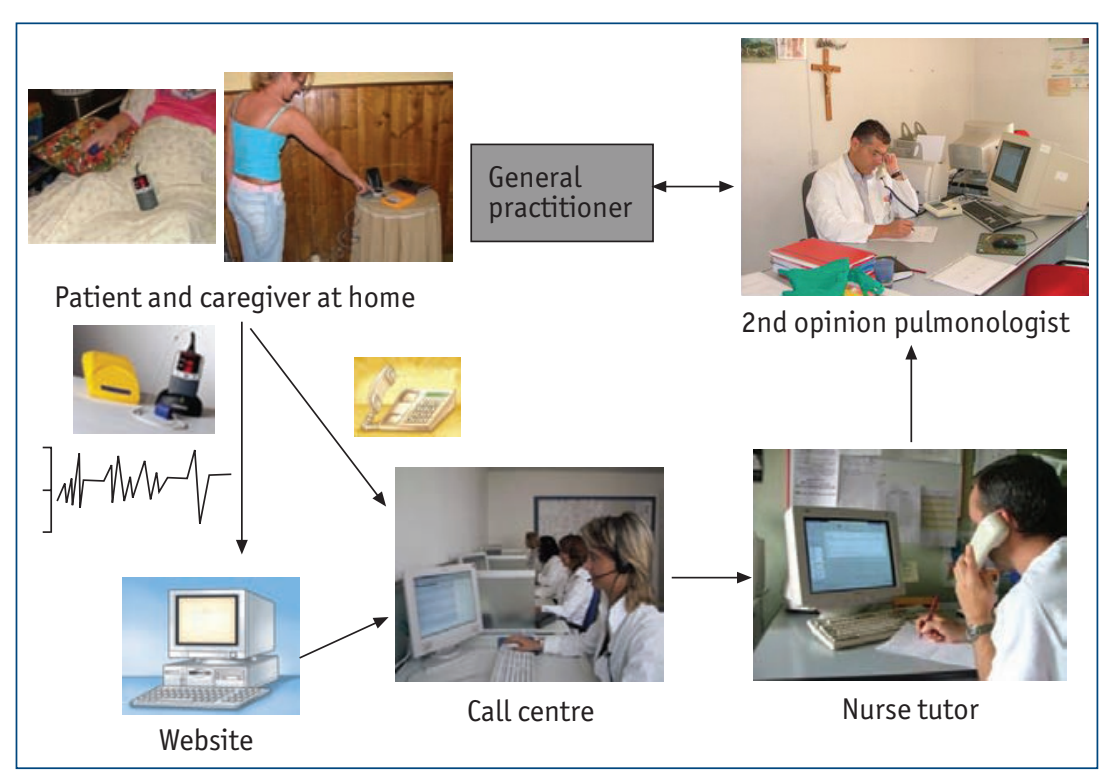




\section{Figure 4}

Study patient during the 2nd day of home weaning protocol. $\mathrm{Sp}, \mathrm{O}_{2}$, $H R$, respiratory rate $(R R)$, minute ventilation $\left(V^{\prime} E\right)$ and airway pressure (Paw) trends from 07:00 to 20:00 $\mathrm{h}$. The figure shows the two steps of the SBT.

\section{Case study 3}

Tentative weaning from MV was carried out in a 63-year-old female at home by means of a telepneumology programme. The programme consisted of telephone follow-up and measurement of $\mathrm{Sp}_{1} \mathrm{O}_{2}$, and breathing pattern trace transmitted via a home telephone line. Heart rate, $\mathrm{Sp}_{1} \mathrm{O}_{2}$, breathing pattern and arterial blood pressure were continuously monitored. The duration of each spontaneous breathing trial (SBT) period was progressively increased starting from 30 minutes up to 8 hours. Under the direct supervision of a pulmonologist, the telephone assistance of the programme's nurse tutor and the aid of the caregiver, the patient was able to maintain diurnal spontaneous breathing after 24 SBT steps (two per day). This case report shows the potential of a structured telepneumology programme in the care and management of respiratory patients, including those on MV.
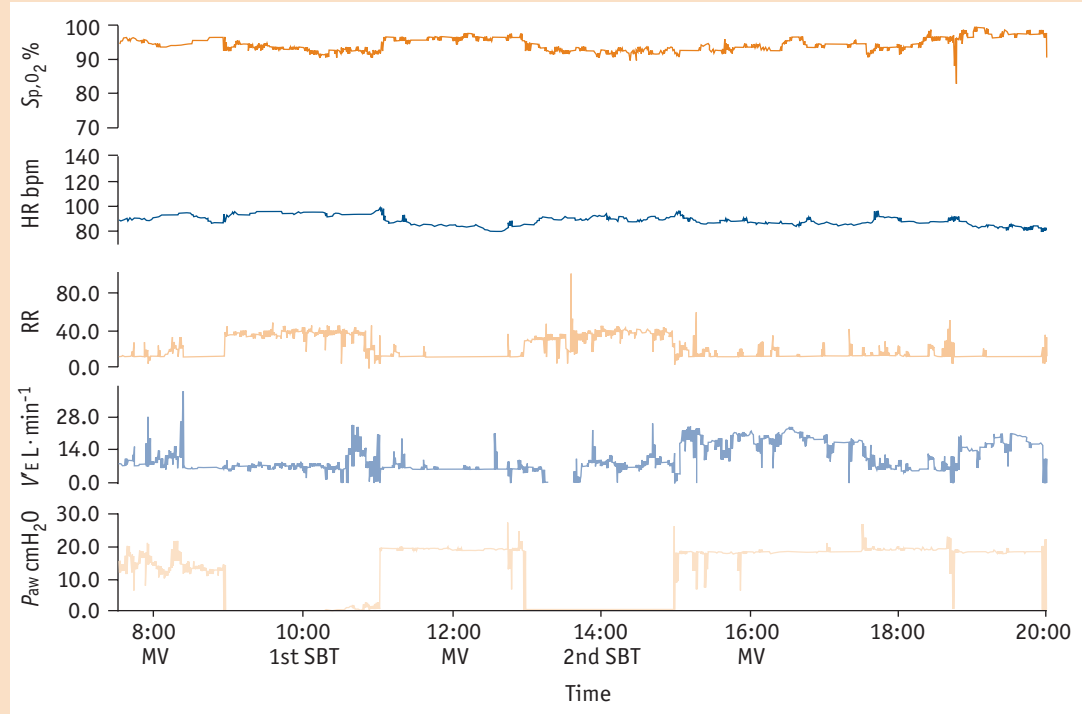

To improve the quality control of home ventilators and patients' requirements, recommendations on the need for some form of follow-up have been addressed [33], in particular using modern technologies of information and

\section{Table 3 Recommendations and limitations for telecare}

\section{Recommendations}

A clearly and articulated mission

Specific goals to be achieved

Accountable governance structure

Well-defined service

Well-defined target population

Well-structured service providers

Detailed procedures and protocols (with continuing quality control)

Correct choice of technology adequate for each situation

Well-structured outcome perspective

Self-sustaining programmes

\section{Limitations}

Many different software, hardware and telecommunication options

Poor specification design for each condition

Poor uniformity for standards

No clear strategies for promoting wide utilisation

Limited technological and clinical research

Legal/confidentiality problems between subjects involved

Costs

Poor knowledge and culture

Scepticism from doctors

Absence of reimbursements communication as remote telemonitoring of ventilation variables, facilitating exchange of information between the different partners involved in home MV. It is well known that MV patients require considerable resources from their healthcare service in terms of hospitalisations, relapses, medical visits, drugs and devices [34], which results in difficult resource allocation decision making. A principal aim of telemedicine is to improve the access to healthcare services, whilst saving time and travel costs. It seems reasonable that more severe patients will need more close follow-up. Telemedicine should be tailored according to the needs and aims of the therapy at various stages: for example, the period just after diagnosis and start of home MV; follow-up after discharge; evaluation of the worsening of slowly progressing clinical conditions; or close follow-up in diseases with very fast deterioration, such as ALS.

A total of 164 consecutive patients attending a busy respiratory outpatient service were asked whether the concept of alternating face-toface consultation with consultation by either telephone or e-mail was acceptable [35]. Over one-third of patients were thought to be suitable for alternating face-to-face with telephone consultation. The diagnoses in these cases included 
asthma, suspected obstructive sleep apnoea, COPD, unexplained cough, and some patients with respiratory malignancy [35]. However, for those with asthma and those awaiting results of investigations especially, telephone consultation appeared to be an acceptable and convenient way of reducing the pressure upon the time available for outpatient consultations. Table 3 summarises the final recommendations and limitations for telecare.

\section{Conclusions}

Patients require suitable healthcare, and this should be balanced with their desire to remain at home for as long as possible. Telehealth enables patients to receive some services at home that were only previously available in hospitals. A principal aim of telemedicine is to improve access to healthcare services. During the process, telemedicine should produce savings in time and travel costs. Besides the advantages of an early diagnosis and a tailored therapeutic management of chronic illnesses in the home, further potential uses of telemedicine include the availability of specialist teleconsultations out of hospital, and improvement in appropriateness of hospital admissions and referrals to the emergency department.

When new technologies are introduced into practice, they are often used without a clear idea of which patients will benefit most, the balance of benefits and harms, and the value for money that technology can offer. While intervention trials discussed here have utilised different designs, the nurse practitioner is common to all the trials and appears to have a key role in the management of chronic disease. The different approaches (multidisciplinary home-based, integrated and telematic) represent the variety of possibilities to be explored in the future. Furthermore, the number of patients that could potentially be involved is enormous. Results on the long-term effect, costeffectiveness analysis, quality of life and public health burden reduction remain to be demonstrated.

Nowadays, a lot of applications of telemedicine are possible and operative. Programmes based on specific and local situations, and on specific diseases and their levels of severity with a high level of flexibility are likely to be the options for reducing care costs. Home telemedicine is not strictly "technology", but an innovative instrument (based on healthcare professionals more than high-tech instruments) that will help the healthcare teams to serve patients and their families, changing the way we deliver care to patients and the relationships between patients and healthcare professionals.

The field of ehealth is relatively new and expanding. In order to establish evidence-based guidelines for the design and implementation of diseasemanagement applications that employ telemedicine and ehealth, further research is required. In the future, it is hoped that telemedicine will form a valuable part of the disease management process for home care, because, when used intelligently, it will supplement conventional delivery techniques but not replace them.

\section{Educational questions}

1. The expected results from a telemedicine programme are:
a) Reduction in hospital stay (days).
b) Reduction in number of calls for GP.
c) To support GP for diagnosis, drug change and follow-up of relapses.
d) All of the previous.
e) None of the previous.

2. Telecare, telemonitoring and telemedicine programmes will change:
a) Modality of hospital access.
b) Relationships between healthcare teams.
c) Methods of drug prescription.
d) All of the previous.
e) None of the previous.

3. Scientific evidence has demonstrated the superiority of telemedicine versus standard follow-up models.
a) True.
b) False.

4. Telemedicine has to be considered as:
a) A new mandatory alternative to hospital admission.
b) A new supplement care instrument to use alongside conventional systems. 


Suggested answers
1. d
2. d
3. b
4. b

\section{References}

1. Wooton R, Dimmick SL, Kvedar JC. Home telehealth: connecting care within the Community. London, The Royal Society Medicine Press Ltd, 2006.

2. Scalvini S, Vitacca M, Paletta L, Giordano A, Balbi B. Telemedicine: a new frontier for effective healthcare services. Monaldi Arch Chest Dis 2004; 61: 226-233.

3. Whitten SP, Mair SF, Haycox A, May RC, Williams TL, Hellmich S. Systematic review of cost effectiveness studies on telemedicine interventions. BMJ 2002; 324: 1434-1437.

4. Mair F, Whitten P. Systematic review of studies of patients satisfaction with telemedicine. BMJ 2000; 320: $1517-1520$.

5. Wootton R. Telemedicine: clinical review. BMJ 2001; 323: 557-560.

6. Riegel B, Carlson B, Kopp Z, Le Petri B, Glaser D, Unger A. Effect of a standardised nurse case-management telephone intervention on resource use in patient with chronic heart failure. Arch Intern Med 2002; 162: 705-712.

7. Harno K, Paavola T, Carlson C, Viikinkoski P. Patient referral by telemedicine: effectiveness and cost analysis of an intranet system. J Telemed Telecare 2000; 6: 320-329.

8. Vassallo DJ, Hoque F, Farquarson Roberts M, Patterson V, Swinfen P, Swinfen R. An evaluation of the first year's experience with a low cost telemedicine link in Bangladesh. J Telemed Telecare 2001; 7: 125-138.

9. Scalvini S, Zanelli E, Domenighini D, et al. Telecardiology community: a new approach to take care of cardiac patients. Cardiologia 1999; 44: 921-924.

10. Wallace $P$, Haines $A$, Harrison $R$, et al. Joint teleconsultation (virtual outreach) versus standard outpatient appointments for patients referred by their general practitioner for a specialist opinion: a randomised trial. Lancet 2002; 359: 1961-1968.

11. Pinnock H, Bawden R, Proctor R, Wolfe S, Scullion J, Price D. Accessibility, acceptability and effectiveness in primary care of routine telephone review of asthma: pragmatic, randomised controlled trial. BMJ 2003; 326: 477-479.

12. Finkelstein J, Cabrera MR, Hripcsak G. Internet-based home asthma telemonitoring: can patients handle the technology? Chest 2000; 117: 148-155.

13. Chan DS, Callahan CW, Sheets SJ, Moreno CN, Malone FJ. An Internet-based store-and-forward video home telehealth system for improving asthma outcomes in children. Am J Health Syst Pharm 2003; 60: 1976-1981.

14. Steel S, Lock S, Johnson N, Martinez Y, Marquilles E, Bayford R. A feasibility study of remote monitoring of asthmatic patients. J Telemed Telecare 2002; 8: 290-296.

15. Feinlieb M, Rosenborg HM, Collins JG, et al. Trends in COPD mortality in the United States. Am Rev Respir Dis 1989; 140: S9-S18.

16. American Thoracic Society. Standards for the diagnosis and care of patients with chronic obstructive pulmonary disease. Am J Respir Crit Care Med 1995; 152: S77-S120.

17. Orient JM. When do patients with chronic obstructive lung disease need hospital admission? Reflections based on a VA experience. South Med J 1983; 76: 593-598.

18. Tougaard L, Krone T, Sorknaes A, et al. Economic benefits of teaching patients with chronic obstructive pulmonary disease about their illness. Lancet 1992; 339: 1517-1520.

19. Haggerty MC, Stockdale-Woolley R, Nair S. Respi-care: an innovative home care program for the patient with chronic obstructive pulmonary disease. Chest 1991; 100: 607-612.

20. Littlejohns P, Baveystock CM, Parnell H, Jones PW. Randomized controlled trial of the effectiveness of a respiratory health worker in reducing impairment, disability, and handicap due to chronic airflow limitation. Thorax 1991; 46: 559-564.

21. Cockcroft A, Bagnall $P$, Heslop A, et al. Controlled trial of respiratory health worker visiting patients with chronic respiratory disability. BMJ 1987; 294: 225-228.

22. Young M, Sparrow D, Gottlieb D, Selim A, Friedman R. A telephone-linked computer system for COPD care. Chest 2001; 119: 1565-1575.

23. Agha Z, Schapira RM, Maker AH. Cost effectiveness of telemedicine for the delivery of outpatient pulmonary care to a rural population. Telemed J E Health 2002; 8: 281-291.

24. Johnston B, Wheeler L, Deuser J, Sousa KH. Outcomes of the Kaiser Permanente Tele-Home Health Research Project. Arch Fam Med 2000; 9: 40-45.

25. Casas A, Troosters T, Garcia-Aymerich J, et al. Integrated care prevents hospitalisations for exacerbations in COPD patients. Eur Respir J 2006; 28: 123-130.

26. Oki Y, Shiomi T, Sasanabe $R$, et al. Multiple cardiovascular risk factors in obstructive sleep apnea syndrome patients and an attempt at lifestyle modification using telemedicine-based education. Psychiatry Clin Neurosci 1999; 53: 311-313.

27. Lankford DA. Wireless CPAP patient monitoring: accuracy status. Telemed J E Health 2004; 10: 162-169.

28. Doolittle GC. A cost measurement study for a home-based telehospice service. J Telemed Telecare 2000; 6: Suppl. 1, S193-S195.

29. Noel HC, Vogel DC, Erdos JJ, Cornwall D, Levin F. VA Connecticut Healthcare Systems, West Haven, Connecticut, USA. Home telehealth reduces healthcare costs. Telemed J E Health. 2004; 10: 170-183.

30. Maiolo C, Mohamed EI, Fiorani CM, De Lorenzo A. Home telemonitoring for patients with severe respiratory illnesses: the Italian experience. J Telemed Telecare 2003; 9: 67-71.

31. Miyasaka K, Suzuki Y, Sakai H, Kondo Y. Interactive communication in high-technology home care: videophones for pediatric ventilatory care. Pediatrics 1997; 99: E1.

32. Vitacca M, Pizzocaro P, Assoni G, et al. A pilot study of nurse-led, home-based telepneumology for 45 patients with chronic respiratory insufficiency with mechanical ventilation assistance. J Telemed Telecare 2006; 12: 337-342.

33. Farre R, Lloyd-Owen SJ, Ambrosino N, et al. Quality control of equipment in home mechanical ventilation: a European survey. Eur Respir J 2005; 26: 86-94.

34. Make BJ, Hill NS, Goldberg AI, et al. Mechanical ventilation beyond the intensive care unit. Report of a Consensus Conference of the American College of Chest Physicians. Chest 1998; 113: 289S-344S.

35. Partridge MR. An assessment of the feasibility of telephone and email consultation in a chest clinic. Patient Educ Couns 2004; 54: 11-13. 